\title{
Successful pregnancies in cows following double freezing of a large volume of semen
}

\author{
Amir Arav*, Yoel Zeron, Haim Shturman, Haim GacituA \\ Institute of Animal Science, Agricultural Research Organization (ARO), \\ The Volcani Center, Bet Dagan, 50250, Israel
}

\begin{abstract}
The objective of the following paper is to describe a new technology for large volume and double freezing of semen in $12 \mathrm{~mL}$ test tubes. Semen from two different bulls was frozen with a new technique using $12 \mathrm{~mL}$ test tubes and was refrozen after thawing in mini straws. All freezing was done in a "Multi thermal gradient" (MTG) freezing apparatus, which moves the container at a constant velocity $(\mathrm{V})$ through a thermal gradient $(\mathrm{G})$ producing a controlled cooling rate $\mathrm{B}=(\mathrm{G}) \times(\mathrm{V})$. Each of the two bulls ejaculated were evaluated for post thaw motility in the lab and then in a field trial which was carried out in a split sample mode. We inseminated 105 cows after a double freezing/thawing cycle, and another 123 cows were inseminated with semen frozen in mini-straws and a conventional method. The results showed a $75 \pm 5 \%$ post thaw motility after freezing a $12 \mathrm{~mL}$ test tube and $50 \pm 5 \%$ after a second freezing/thawing in mini-straws, respectively. Controlled vapour freezing showed a $60 \pm 10 \%$ post thaw motility. The results of the field trial showed a pregnancy rate of $44 \%(47 / 105)$ for the double freezing group in comparison to $45.5 \%(56 / 123)$ for the controlled group. These results can be beneficial for large volume freezing, and therefore for bull semen cryobanking in a large volume which will be followed by second freezing in a regular insemination volume.
\end{abstract}

freezing / cryopreservation / cryobanking / sperm / bull

\section{BULL SEMEN CRYOBANKING}

Cryobanking of semen has had a major impact on dairy cattle genetic breeding [2] In addition to its role in young bull genetic breeding, cryobanking of bull semen is an important backup for sufficient insemination doses in cases of disease, infertility or mortality.

* Correspondence and reprints

E-mail: arav@agri.huji.ac.il
Freezing and storage of semen is done regularly using mini $(1 / 4 \mathrm{cc})$ or midi $(1 / 2 \mathrm{cc})$ straws. However, cryobanking of a large number of straws is time consuming, expensive and requires a lot of storage space and liquid nitrogen. An alternative procedure which will reduce these expenses could be the freezing of a whole ejaculate in one test tube $(12 \mathrm{~mL})$ and only when needed (when 
the bull is a "proven bull"), the test tube will be thawed and will then be refrozen in regular mini straws. We describe here the use of a new technology for large volume (whole ejaculate) freezing/thawing and refreezing in mini-straws.

\section{MTG TECHNOLOGY}

Our novel freezing technology is based on "Multi-thermal gradient $\left(\mathrm{MTG}^{\mathrm{R}}\right.$, IMT, Israel) [1]" directional solidification and is used mainly for freezing sperm and large tissue. The semen in the test tube is moved at a constant velocity $(\mathrm{V})$ through a linear temperature gradient $(\mathrm{G})$ so the cooling rate $(\mathrm{G} \times \mathrm{V})$ and ice front propagation are precisely controlled (Fig. 1).

This method also enables the incorporation of controlled seeding into the freezing process. When any liquid is cooled below its freezing point, it remains a liquid, in an unstable super-cooled state, until freezing starts at randomly distributed nucleation sites and spreads throughout the entire volume of the liquid. As discussed above, in the conventional equiaxed method of freezing, ice grows at an uncontrolled velocity and morphology, and may disrupt and kill the cells of the samples. Ideally, the velocity of the freezing front should be such that the ice morphology does not disrupt the cells or tissue. However, the rate of cooling appropriate for favourable ice morphology may not be appropriate for other desired outcomes of a sample's freezing protocol. The laterally varying gradient used in our technology allows cooling to proceed at differing rates under varied temperature regimes, thereby facilitating full control over nucleation and ice crystal morphology. This technique allows very precise control of the cooling rate $\left(0.01\right.$ to $\left.1000{ }^{\circ} \mathrm{C} \cdot \mathrm{min}^{-1}\right)$ within a large volume.

The MTG apparatus can control ice crystal propagation by changing the thermal gradient $(\mathrm{G})$ or the liquid-ice interface velocity (V) and so optimizing the ice crystal morphology during freezing of cells and tissue. The rate of cooling also affects the morphology of the intercellular ice freezing [5] and also the morphology of the intracellular ice crystals: morphologies such as closely

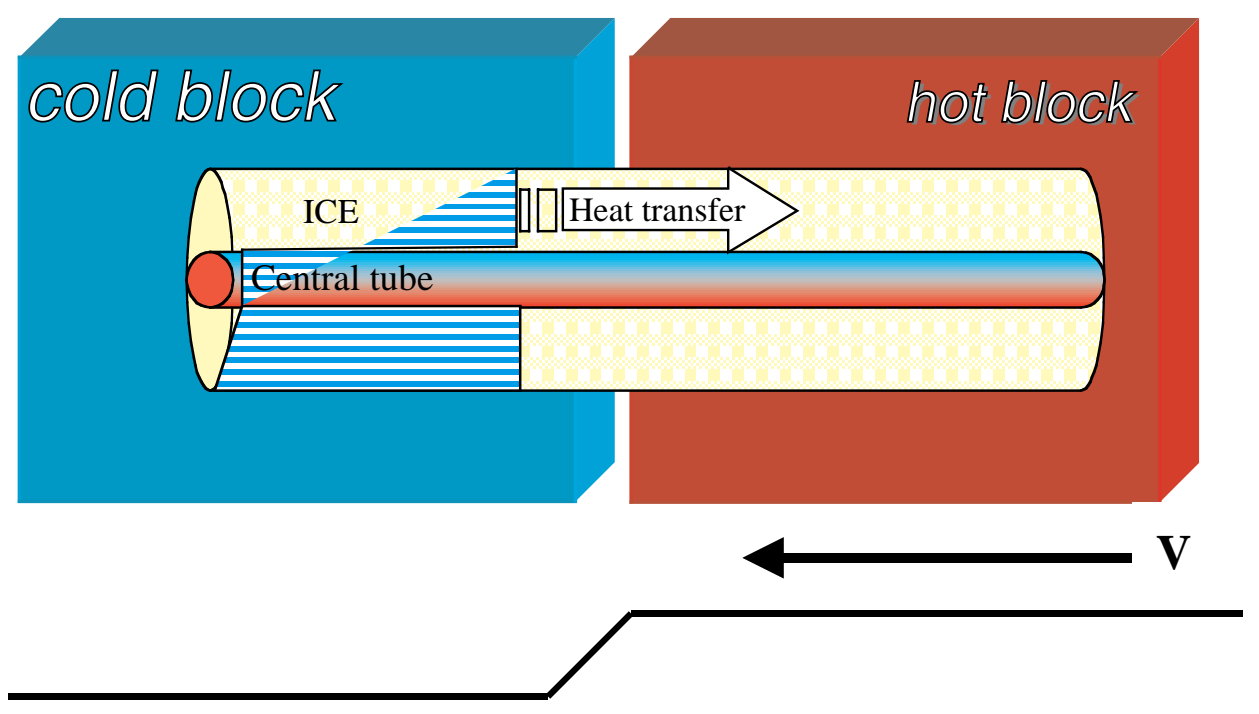

Figure 1. Schematic design of the MTG freezing apparatus. 


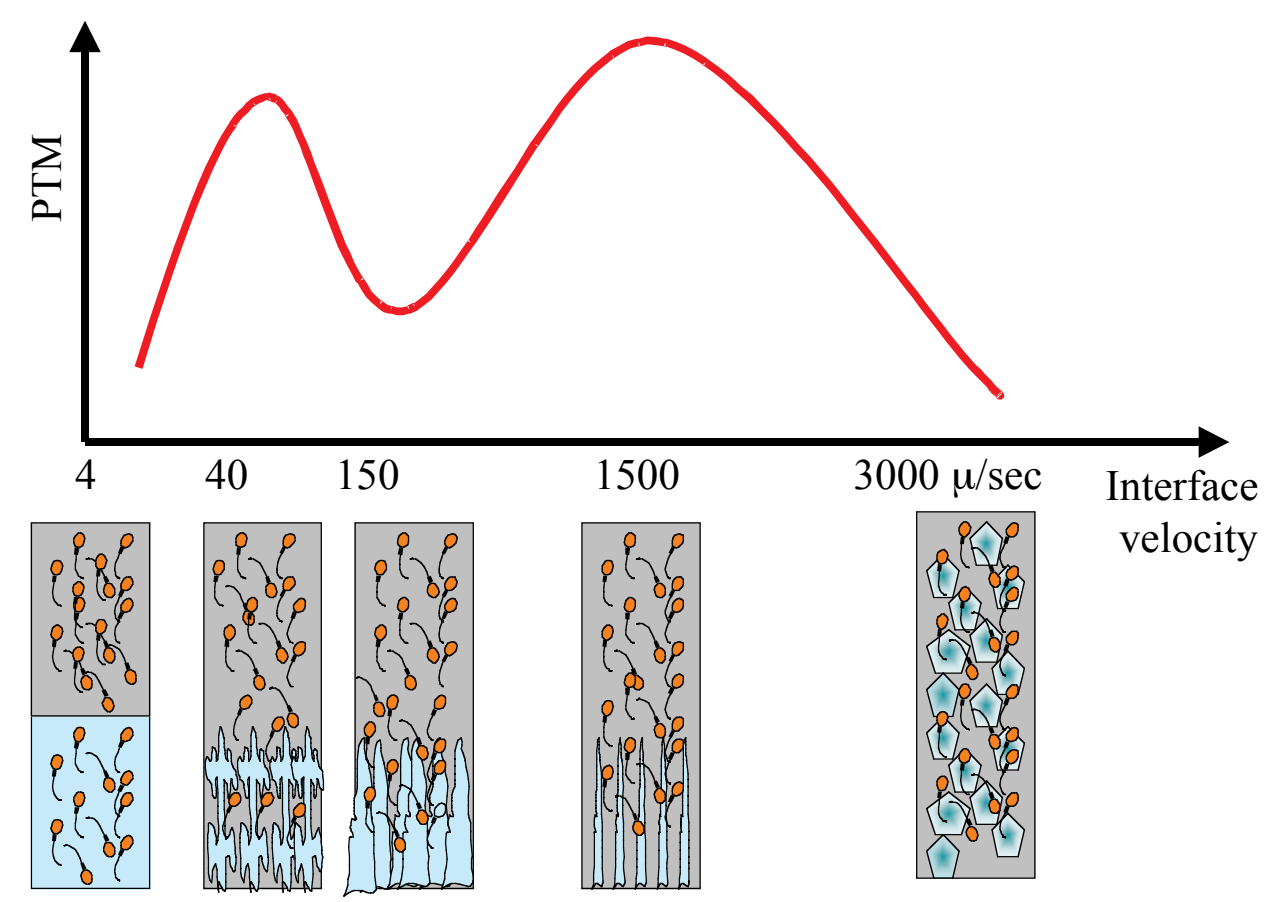

Figure 2. Effect of interface velocity (V) on ice crystal morphology and sperm post thaw motility (PTM).

packed needles kill cells by external mechanical damage (unpublished observation). Thus, maximising the survival rate of cells subjected to freezing and thawing requires careful control of the freezing process i.e. interface velocity. Using a cryomicroscopy observation, we found that the survival of sperm shows a biphasic curve where at a very slow velocity ice will grow in a planner form which will kill all cells. At a higher velocity, ice crystals will form secondary branches and survival will increase, however at an even higher velocity (i.e. $300 \mu \mathrm{m} \cdot \mathrm{s}^{-1}$ ) ice will start to form "needle-like" ice crystals which will decrease PTM, but at an even greater velocity, will permit very high survival (Fig. 2) depending on the space between the ice crystals [6]. Finally, at an extremely high velocity (i.e. $>3000 \mu \mathrm{m} \cdot \mathrm{s}^{-1}$ ), directional solidification will not occur and survival will decrease.

\section{HEAT TRANSFER PROBLEMS ASSOCIATED WITH LARGE VOLUME FREEZING}

In a conventional slow-freezing method, the temperature of the chamber is dropped in a controlled stepwise manner. This method is based on using multidirectional (equiaxed) heat transfer to achieve a rate of temperature change in the sample that depends on the thermal conductivity and geometrical shape of the container and of the biological material within it [4]. The thermal gradient within the sample is determined implicitly by the temperature of the chamber and the thermal conductivity of the materials of the sample, and is not directly controllable. Furthermore, the ambient temperature gradients within the freezing chamber and the unreliability of temperature recording measurements [3] add to the difficulty of 
achieving the optimal cooling rate in a large volume sample.

\section{CRYOBANKING OF LARGE VOLUME SEMEN}

Each ejaculate $(n=3)$ was tested for semen concentration and motility $(>70 \%)$ before dilution. We used AndroMed ${ }^{\circledR}$ (minitub, Hauptstrabe, Germany) for the semen dilution to a final concentration of $15 \times 10^{6} \mathrm{sperm} \cdot \mathrm{mL}^{-1}$.

Freezing of a whole ejaculate was done in a special test tube $(12 \mathrm{~mL})$. Heat transfer was opposite to the test tube movement and was parallel to the tube length axis (Fig. 1).

Sperm post thaw mobility (PTM) after freezing in a large volume was very high. We found a survival rate of $90-100 \%$ (normalised PTM) in the two bulls we cryopreserved in the MTG technique. These results were superior to MTG freezing using mini straws (data not shown), which suggest the benefit of using MTG freezing with a large volume for sperm cryopreservation. The results showed a $75 \pm 5 \%$ post thaw motility after freezing a $12 \mathrm{~mL}$ test tube and $50 \pm 5 \%$ after second freezing/thawing in mini-straws, respectively. Controlled vapour freezing showed a $60 \pm 10 \%$ post thaw motility which was lower than the results after MTG freezing of mini straws.

The results of the field trial showed a pregnancy rate of $44 \%(47 / 105)$ for the double freezing group in comparison to $45.5 \%$ $(56 / 123)$ for the controlled group.

The large freezing volume may be very useful for cryobanking of bull semen, for example, at AI centres which have a bank of 10000 straws made from 25 ejaculates (400 straws/ejaculate). We calculated that these 10000 straws will fit into 13 goblets (750 straw/goblet). In comparison, when we freeze a large volume ( $12 \mathrm{~mL}$ test tube) the 25 ejaculates will be frozen in 25 test tubes which will be stored only in 2 goblets. This means that we need 6.5 times more goblets using straws in comparison to test tube freezing. In this case, the present method gives a capability to have a bank of "waiting bulls" in some of the AI centers which presently do not use semen cryobanking. In addition this method will save money in labour and consumables (filling, printing, LN for freezing and for storage, etc.).

\section{CONCLUSIONS}

The MTG technique could be very useful for large volume cryopreservation and double freezing. The ability to control the ice crystal morphology, the homogeneous cooling rate through a large volume sample and the continual seeding during the whole freezing process makes it possible to freeze a large volume without compromising sperm viability.

Cryobanking of whole ejaculates of bull semen has many benefits compared to freezing of numerous straws. It will be possible to save all expenses related to freezing and storage. In addition, large volume freezing might be a benefit in other species such as the stallion and boar.

\section{REFERENCES}

[1] Arav A., Device and methods for multigradient directional cooling and warming of biological samples, US Patent (1999) 5,873,254.

[2] Foote R., Park J., Factors affecting preservation and fertility of bull sperm: a brief review, Reprod. Fertil. Dev. 5 (1993) 665-673.

[3] Karlasson J.O.M., Toner M., Long term storage of tissues by cryopreservation: critical issues, Biomaterials 17 (1996) 243-256.

[4] Koebe H.G., Werner A., Lange V., Schildberg F.W., Temperature gradients in freezing chambers of rate-controlled cooling machines, Cryobiology 30 (1993) 349-353.

[5] Mazur P., The role of intracellular freezing in the death of cells cooled at supraoptimal rates, Cryobiology 14 (1977) 251-272.

[6] Mazur P., Cole K.W., Role of unfrozen fraction, salt concentration, and changes in cell volume in the survival of frozen human erythrocytes, Cryobiology 1 (1989) 1-29. 